\title{
Consumers' choice-blindness to ingredient information
}

\author{
T.T.L. Cheung a, *, 1 , A.F. Junghans ${ }^{\text {a, }}{ }^{*}, 1$, G.B. Dijksterhuis ${ }^{\text {b }}$, F. Kroese ${ }^{\text {a }}$, P. Johansson ${ }^{\text {c }}$, \\ L. Hall ${ }^{\mathrm{c}}$, D.T.D. De Ridder ${ }^{\mathrm{a}}$ \\ a Department of Psychology, Utrecht University, Heidelberglaan 1, 3508TC, Utrecht, The Netherlands \\ ${ }^{\mathrm{b}}$ Sensory and Consumer Science Section, University of Copenhagen, Copenhagen, Denmark \\ ${ }^{\mathrm{c}}$ Lund University Cognitive Science, Lund, Sweden
}

\section{A R T I C L E I N F O}

\section{Article history:}

Received 26 May 2015

Received in revised form

11 August 2015

Accepted 17 September 2015

Available online 25 September 2015

\section{Keywords:}

Choice-blindness paradigm

Food choice

Ingredient information

Attention

Clean label

\begin{abstract}
A B S T R A C T
Food manufacturers and policy makers have been tailoring food product ingredient information to consumers' self-reported preference for natural products and concerns over food additives. Yet, the influence of this ingredient information on consumers remains inconclusive. The current study aimed at examining the first step in such influence, which is consumers' attention to ingredient information on food product packaging. Employing the choice-blindness paradigm, the current study assessed whether participants would detect a covertly made change to the naturalness of ingredient list throughout a product evaluation procedure. Results revealed that only few consumers detected the change on the ingredient lists. Detection was improved when consumers were instructed to judge the naturalness of the product as compared to evaluating the product in general.

These findings challenge consumers' self-reported use of ingredient lists as a source of information throughout product evaluations. While most consumers do not attend to ingredient information, this tendency can be slightly improved by prompting their consideration of naturalness. Future research should investigate the reasons for consumers' inattention to ingredient information and develop more effective strategies for conveying information to consumers.
\end{abstract}

() 2015 Elsevier Ltd. All rights reserved.

\section{Introduction}

When it comes to food products, many consumers often report preferring natural products (Rozin et al., 2004), and assume that products based on natural ingredients without additives are healthier (Bredahl, 1999; Dickson-Spillmann, Siegrist, \& Keller, 2011; Evans, de Challemaison, \& Cox, 2010; Shim et al., 2011). In response food manufacturers have spent substantial efforts in tailoring the presentation of ingredient list information on food packaging with the underlying assumption that consumers infer the 'naturalness' of a food product by its ingredients. Similarly, policy makers have increasingly focused on providing objective information about the naturalness of ingredients in food products. Nonetheless, the effect that ingredient list information has on consumers remains unclear,

\footnotetext{
* Corresponding authors.

E-mail addresses: t.t.l.cheung@uu.nl (T.T.L. Cheung), a.f.junghans@uu.nl (A.F. Junghans), garmtdijk@gmail.com (G.B. Dijksterhuis), f.m.kroese@uu.nl (F. Kroese), petter.johansson@lucs.lu.se (P. Johansson), lars.hall@lucs.lu.se (L. Hall), d.t.d.deridder@uu.nl (D.T.D. De Ridder).

1 Equal contributions.
}

as there is a lack of scientific evidence demonstrating that consumers actually prefer products with more 'natural' ingredients. Accordingly, the first objective of the current study is to examine the degree to which consumers take the initial step to actually attend to ingredient information on food packaging. Contrasting the previously employed self-report measures, the novelty of this study is the employment of the choice-blindness paradigm (Johansson, Hall, Sikstroöm, \& Olsson, 2005) to investigate whether consumers pay attention to ingredient information on product packaging. Given consumers' limited attention to product labels (Grunert, Wills, \& Fernández-Celemin, 2010), we furthermore explore whether the provision of subtle reminders could encourage consumers' attention to ingredient lists. By investigating the effectiveness of reminders to consider naturalness, the current findings are relevant for both policy makers and food manufacturers' efforts in enhancing consumers' consideration of ingredient list information.

\subsection{Favoring 'natural' over 'unnatural' ingredients}

While consumers report having a preference for more natural food (Rozin et al., 2004), it is unclear whether they actively seek out 
information to evaluate the 'naturalness' of different food products. Existing literature has mainly focused on examining consumers' use of ingredient list information on packaging for nutritional value (see Grunert \& Wills, 2007 for review), but not for deducing the naturalness of food products. In order to address this research gap, the current research adopts a novel approach by examining consumers' consideration of E-numbers on ingredient lists of food packaging. Enumbers, which are reference numbers given to identify food additives in the EU, (e.g., pectin is a gelling agent that is commonly used in jam and identified by the code E440), is a topic highly discussed in contemporary media and public discourse, as it captures the increasing trend amongst consumers for more 'natural' food products and concerns over food additives, as well as the responses of food authorities and food manufacturers (Evans et al., 2010).

While E-numbers were initially designed by the European Food Safety Authority to identify all food additives that have been extensively tested against potential health risks (Van Dillen, Hiddink, Koelen, de Graaf, \& van Woerkum, 2003), ironically, consumers often associate them with undesirable, harmful, and unhealthy chemicals (Evans et al., 2010; Hoogenkamp, 2012; McCarthy et al., 2007; Varela \& Fiszman, 2013). Moreover, despite previous findings show that only a minority of consumers look at food labels for nutritional information (Grunert et al., 2010), manufacturers have been increasingly pushing for clean label products (Bobe \& Michel, 2011; Hoogenkamp, 2012), which are defined by being free of 'chemical' additives, having easy-to-understand ingredient lists, and being produced by use of traditional techniques with limited processing (Edwards, 2013). Indeed, between 2003 and 2012 the number of products with such clean labels has more than quadrupled universally (Edwards, 2013). In spite of all the initiatives taken to satisfy consumers' seemingly growing preference for more natural products, there is a pressing need for scientific evidence to justify these initiatives.

\subsection{The validity of self-report measures}

Previous studies have indeed reported negative attitudes towards additives and E-numbers (Drichoutis, Lazaridis, \& Naygar Jr., 2006; Edwards, 2013; Holm \& Kildevang, 1996), but the majority of these studies are based on self-report measures. There are of course observational studies investigating how consumers use information on packaging, yet these studies have focused on front of package or nutrition value information rather than ingredient lists that provide information on the naturalness of the ingredients (Grunert, Fernandez-Celemin, \& Wills, 2010). However, self-report measures have been criticized for being vulnerable to task demands and social desirability influences, which result in low predictive power of reported attitudes for actual behavior (Azjen \& Fishbein, 2005; Hebert, Clemow, Pbert, Ockene, \& Ockene, 1995; Vermeir \& Verbeke, 2006). Previous research has shown that, particularly in the realm of health, responses are assimilated towards the socially desired answer (Herbert et al., 1995; Klesges et al. 2004; Kristiansen \& Harding, 1984) due to people's motivation to consider and present themselves as healthy individuals (Bailis, Segall, \& Chipperfield, 2003; Lindeman \& Stark, 1999; Malhotra, 1988). As such, using self-report measures that require participants to provide opinions to topics they do not have stable opinions about further increase the influence of strongly negative discourse, such as the media attention to food additives that has mostly framed food additives in terms of risks involved in consuming additives and the contamination of an otherwise natural product (Evans et al., 2010), to bias opinions and preferences (Reed II, Wooten, \& Bolton, 2002; cf. Dijksterhuis, 2004). Consequently, when opinions are spontaneously formed under the influence of such external sources it is not surprising that the resulting opinions do not correspond with behavior.
These issues suggest that product evaluations may depend on whether consumers are specifically asked about whether unnaturalappearing ingredients in the product are appreciated (i.e. where the consumer is directly pointed at the fact that the naturalness is the key factor in the evaluation) or whether consumers are asked to evaluate a product that comes with ingredient information but without the trigger to judge the product on its naturalness. For example, as shown by the study by Noussair, Robin, and Ruffieux (2001), self-reported negative attitudes toward genetically modified food did not translate into decreased purchasing of genetically modified food. On one hand, part of this lacking association could be explained by influences on the self-reports in terms of demand characteristics, social desirability, and self-concepts as discussed earlier. On the other hand, it may be that consumers genuinely hold concerns with genetically modified food, but at the actual point of purchase these negative perceptions and attitudes are not acted upon.

Accordingly, the current study aims to overcome these shortcomings of self-report assessments by firstly avoiding the direct reporting of attitudes on E-numbers and by manipulating the degree to which participants are guided towards including naturalness as a factor in their product evaluations. In order to achieve these ends the choice blindness paradigm is used in the current study.

\subsection{The choice-blindness paradigm}

It has recently been shown that people often fail to detect a mismatch between a previously expressed attitude and a (different) attitude they are subsequently presented with as their own, a phenomenon known as choice-blindness (Johansson et al., 2005). In this research paradigm participants are asked to make choices but are subsequently presented with the rejected option as being their selected option. Interestingly, participants often not only fail to detect the mismatch between their initial, actual choice and the presented choice, but they spontaneously confabulate reasons for having made the presented (never made) choice. The lack of detection of such a mismatch has been shown on various dimensions, such as attractiveness of faces, in which participants choose a more attractive face, and are subsequently asked to justify their choice of the originally not chosen other face (Johansson et al., 2005); product preference, in which participants firstly, do not detect a swap of their chosen product and, secondly, confabulate reasons for having chosen the product they never actually chose (Hall, Johansson, Tärning, Sikström, \& Deutgen, 2010); as well as moral and political attitudes (Hall, Johansson, \& Strandberg, 2012; Hall et al., 2013). To illustrate a few examples of the low detection rate, from the aforementioned studies participants only concurrently detected $13 \%$ of the trials in which their chosen face had been changed (Johansson et al., 2005), demonstrated a 33\% detection rate when the unchosen product was returned (Hall et al., 2010), and correctly identified $41 \%$ of the trials when their moral attitude ratings had been manipulated (Hall et al., 2012).

While these previous studies were designed to examine the stability of choices and attitudes, the current study employs the choice-blindness paradigm to investigate the attention to ingredient lists and its importance for product evaluation while overcoming the above-mentioned disadvantages of self-report assessments. The choice-blindness paradigm allows us to infer the degree of attention that is paid towards ingredient lists by presenting the participants with the supposedly same physical product, while in fact changing the ingredient information on the product. We infer that the participant would need to have initially looked at the ingredient list and processed the information to some sufficient degree before they could notice the discrepancy and detect the change on the manipulated ingredient list presented later on in the experiment.

Capturing these advantages of the choice-blindness paradigm, 
the study provides insights into the degree to which the design of more natural products and the accompanying presentation of more natural ingredient lists actually facilitate consumer preference for the more highly valued 'natural' products. It provides a measure to infer whether consumers pay attention to ingredient lists during actual product evaluations. In addition, we explore the possibility that a reminder, in the form of a subtle instruction for consumers to explain their naturalness evaluation of product, could increase the likelihood for consumers to attend to ingredient information on the package, thereby mitigating the choice blindness effect if the ingredient information on the packaging of a food product was changed.

\section{Design and hypotheses}

Accordingly, the current study employs the choice-blindness paradigm of Hall et al. (2010) and adopts a 2 (instruction: general vs. specific) $\times 2$ (ingredient list: no change vs. change) between subjects factorial design. The dependent variable of interest, whether participants detect the change to the ingredient list or not (i.e., online detection vs. no detection), is a categorical outcome.

During the experiment, participants were first instructed to evaluate two products carefully. Subsequently, participants were returned with the product that had received a higher general evaluation rating and were instructed to explain their evaluations based on either the general instruction to justify the general rating or the specific instruction to justify specifically the naturalness rating of the preferred product. In the ingredient list change condition, unbeknownst to the participant, the experimenter swapped the product that the participant had given higher overall rating to with another product that was identical in all aspects of packaging except with a changed ingredient list. Considering that the only way that the participant would have noticed the changed ingredient list on the returned product was if they had initially paid attention to the ingredient list on the product that they had previously evaluated, the detection of such change was used as indicator for attention to ingredient lists.

Based on the detection rates found in previous studies using the choice blindness paradigm, it was expected that few participants would detect the change to the ingredient list information. However, it was expected that the detection rate would be higher in the specific instruction condition, in which participants were asked to explain their naturalness rating compared to the general instruction condition in which participants were asked to explain their overall rating of the product.

In summary, the aim of the choice paradigm used in the current study is to demonstrate consumers' inattention to ingredient list information that contributes to their blindness to change to the ingredient list. Rather than focusing on what consumers provide or confabulate as reasons for their evaluation of the product, the instruction to explain the general evaluation rating or the specific naturalness rating of the product was simply used as a manipulation to facilitate attention towards the ingredient list information as means to mitigate choice blindness. As such, the choice blindness paradigm aims to reveal which information that consumers attend to (or not), and how to increase attention to relevant information through the form of instructional reminders.

\section{Method}

\subsection{Participants}

Participants $(N=534)$ were recruited via a marketing research agency for monetary reward. All participants were residents in the Netherlands and capable of the Dutch language. Forty-two participants were excluded from the analysis due to not following the procedures and providing insufficient data. The final dataset consisted of 492 participants; 37.4\% were in the ingredient list no change condition and $62.6 \%$ were in the ingredient list change condition. Participants included 53\% females and $46.5 \%$ males (remaining .5\% did not disclose their gender) with a mean age of 39 years $(S D=14.17)$. Educational levels ranged from $2.7 \%$ with basic educational, 55.3\% vocational training and higher secondary education, and $42 \%$ with university degrees. At the time of the study $28.8 \%$ were unemployed and $71.2 \%$ were employed.

The study was conducted in accordance with the ethical standards described by the Medical Research Involving Human Subjects Act (WMO, 2012), according to which research with healthy adults is exempted from the requirement for formal ethical approval. The study was conducted by OP\&P Product Research in accordance with ESOMAR code (ESOMAR, 2015).

\subsection{Procedure}

Participants were invited to the marketing research agency to take part in a marketing study on soup. They were randomly assigned to one of four conditions. Upon arrival participants were greeted by the hypothesis-blind experimenter and guided into an experimentation room where they were asked to sit at a table where two cans of soup were presented next to each other, along with a product evaluation questionnaire for each product. The products included a can of soup from the brand Wouda and the brand Stijn, two entirely fictitious brands which were specifically designed for the present study (the presentation on left and right was counterbalanced). Both products had either 'unnatural' (elaborated descriptions of ingredients with words and E-numbers) or 'natural' (few word descriptions of ingredients) ingredient lists presented on the backside of the can. Further information regarding the overall packaging of the soup cans and the precise differences between the natural vs. unnatural ingredient lists are provided in the Materials section.

The choice blindness paradigm commenced, and in the first stage participants were encouraged to closely examine both products in order to fill out the product evaluation forms. After the participant has completed the evaluation, the experimenter removed the products and the product evaluation forms from the table. The experimenter then presented the participant with a demographic questionnaire to complete.

At the second stage, the experimenter implemented the experimental manipulation. While the participant was filling out the demographic questionnaire, the experimenter examined participants' product evaluation forms and selected the brand that scored higher on the overall general evaluation rating. Critically, the experimental manipulation where the ingredient list changed (in the ingredient list change condition) or remained the same (in the no change condition) was performed on the brand of soup receiving the higher overall rating. In cases where both products had the same overall rating, the experimenter chose either one of the products to use for the remainder of the experiment but ensured that this choice was counterbalanced between participants (Stijn: 104; Wouda: 114).

After the participant had completed the demographic questionnaire, the participant was returned back with the brand of soup that they had given the higher overall rating to (or one of the brands chosen by the experimenter due to equal ratings) along with the product evaluation form that was previously filled out. Presenting the evaluation form again allowed the participant to see the overall and naturalness rating that they had previously assigned to that brand of soup that was returned back to them. At this point of the experiment, the participant was presented back either with a 
can of soup containing the same ingredient list (no change condition), or a can of soup with a different ingredient list (ingredient list change condition) from the product that they had initially evaluated at the first stage of the experiment. To illustrate, in the control no change condition if the participant had initially rated the unnatural ingredient lists, they were handed their preferred brand with the unnatural ingredient list and likewise for the natural ingredient list. In this no change condition, the ingredient list evaluation order was counterbalanced between natural to natural, and unnatural to unnatural. Contrarily, in the ingredient list change condition, participants were returned with a product that was identical in packaging to the product that they had previously assigned a higher overall rating to, but with a changed ingredient list. For instance, had participants previously given a product with an unnatural ingredient list a higher overall rating, they were handed back an identical product but with a natural ingredient list. Or if they had previously given a product with natural ingredient list with a higher overall rating, they were handed back an identical product but with an unnatural ingredient list. The ingredient list change manipulation was counterbalanced between natural to unnatural, and unnatural to natural. The precise differences between the experimental condition in which the soup cans (in essence where ingredient list evaluation orders) changed and the control condition in which the soup cans did not change are illustrated in Fig. 1.

Subsequently, at the third and last stage of the choice-blindness paradigm, the experimenter assessed for change detection by asking the participant to explain why they had given the product the respective score on the overall rating question (general instruction condition) or on naturalness (specific instruction condition), while referring to this score on the product evaluation form. Afterwards, the experimenter removed all the materials and provided the participant with a tablet computer to fill in the final questionnaire.

Had the participant detected the swap of ingredient lists in the experimental condition this was coded as an 'online' detection (detection level code 1), in which case the participant was asked to fill in the final questionnaire and was thanked for their participation. All participants who had not detected a swap online went through a series of detection assessment questions at the end of the experiment. If the participant voiced any detection of the swap following one of these questions, this was coded as follows: The experimenter first asked whether the participant had any questions or comments about the study (detection level 2); whether they had noticed anything during the experiment (detection level 3); and whether they had noticed anything about the products they had evaluated (detection level 4). Finally, participants were thanked and guided toward the exit. Debriefing about the manipulation and aim of the study was done in written form subsequent to the finalization of data collection.

The duration of each experimental session was approximately 10-15 min. Each experimental session was conducted with each participant individually. The experimenter remained in the same room as the participant for the entire duration of the experiment, and whenever the participant was filling out questionnaires (i.e., evaluations of the two soups, demographic questionnaires, and final questionnaires), the experimenter remained in the same room but was not in the immediate vicinity of the participant so he or she could complete the questionnaires discretely.

\subsection{Materials}

\subsubsection{Soup can packaging}

As previously mentioned, the two brands of soup used in the current experiment, Wouda and Stijn, were fabricated specifically for the purpose of the study. The soup packaging was designed respectively for the two brands (see Fig. 2). The soup cans used in the study had a dimension of $12 \mathrm{~cm}$ in height and $10 \mathrm{~cm}$ in diameter.

\subsubsection{Natural vs. unnatural ingredient lists}

The natural and unnatural ingredient lists were initially pretested with 40 participants rating a long vs. a short ingredients list naturalness and healthiness on 10 -point scales $(1=$ not at all natural/healthy to $10=$ very natural/healthy). Pre-test results indicated that the short ingredient list was perceived to be significantly more natural $(M=8.6 ; S D=1)$ than the long ingredient list $(M=3.5$; $S D=1.7) ; t(39)=15.52, p<.001$. The short ingredient list was also perceived as significantly healthier $(M=7.74 ; S D=1.37)$ than the long ingredient list $(M=4.9 ; S D=1.7) ; t(39)=7.53, p<.001$. Based on these pretest results the short ingredient list was used as the 'natural' ingredient list and the long ingredient list was used as the 'unnatural' ingredient list in the experiment (see Fig. 3a and b).

\subsection{Measures}

Throughout the experiment participants were asked to fill out three questionnaires.

\subsubsection{Product evaluation forms}

Participants were asked to evaluate the two presented products based on two product evaluation forms; one for brand Wouda and one for brand Stijn. These questionnaires included evaluations of the products in terms of healthiness, expected tastiness, naturalness, authenticity, familiarity, appeal, liking of the package, the amount to which this product is consumed (this question was often misinterpreted by participants to ask for how often any soup is consumed; consequently, the question was excluded from the analysis); and overall rating. All these questions were answered on 10-point Likert scales. A sample of the product evaluation form could be found in the Appendix.

\subsubsection{Demographic questionnaire}

This questionnaire assessed age, gender, level of education, number of people living in their household, employment status, nationality, and how often participants do grocery shopping (ranging from never to every day on a 5-point scale).

\subsubsection{Final questionnaire}

The final questionnaire assessed participants' concern for health, their typical use of sources of information on product packages, as well as current levels of stress and hunger.

\subsubsection{Justification scores}

Based on the detection assessment participant were categorized as online detectors (detection level 1) if they noticed the swap of the ingredient lists during the experiment; as retrospective detectors if they referred to the swap of ingredient lists during the detection assessment (detection level 2, 3, and 4), and were categorized as non-detectors if they did not notice the swap at all. An additional measure of whether participants mentioned the ingredient lists during justification for their previously given overall ratings or naturalness ratings was recorded.

\section{Results}

\subsection{Randomization check}

There were no significant differences between participants in the general and specific instruction condition in terms of age, gender, educational level, and employment (all $p$ 's > .16). Similarly, 
Control Condition

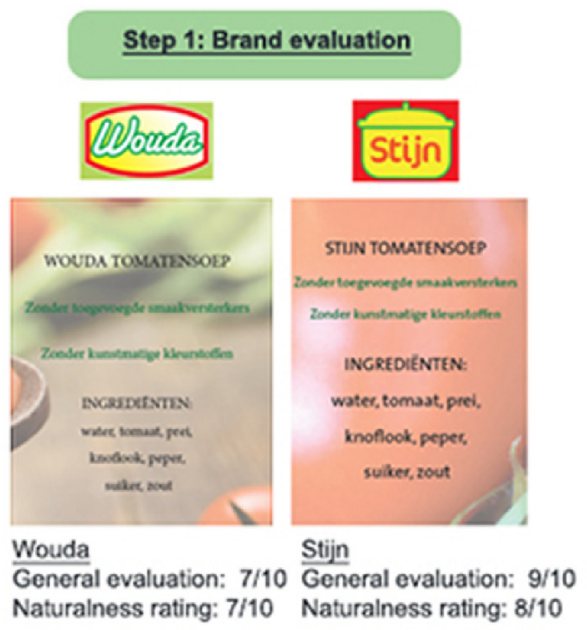

Step 2: Product returned

The brand of soup with the higher general evaluation rating retumed back to the participant
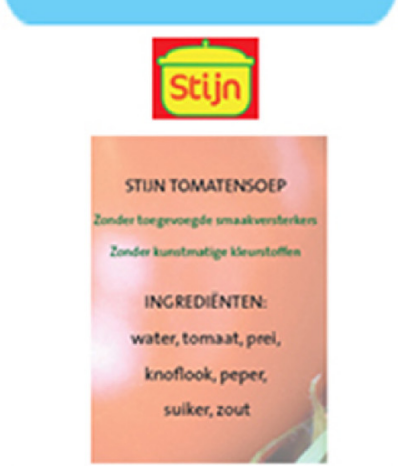

Step 3: Justification of rating

General Instruction: Why did you give this product a general evaluation rating of 9 ?

Or

Specific instruction: Why did you give this product a naturalness rating of 8 ?
Ingredient List Change Condition
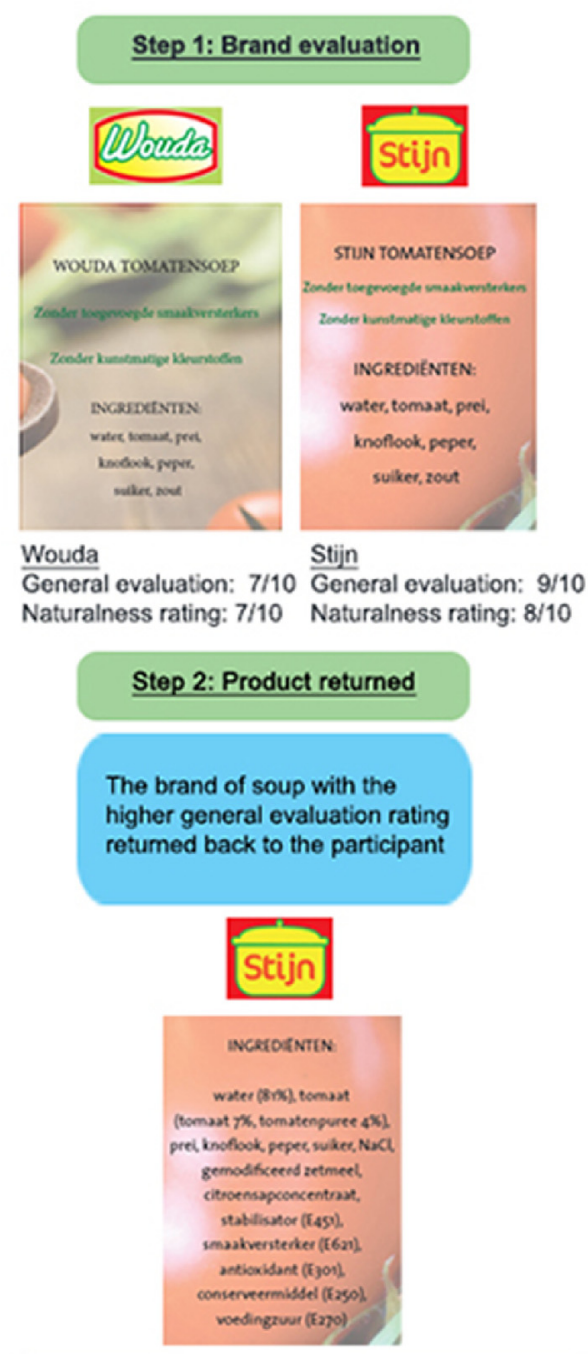

Step 3: Justification of rating (Change detection)

General Instruction:

Why did you give this

product a general

evaluation rating of 9 ?

Or

Specific instruction:

Why did you give this

product a naturalness rating

of 8 ?

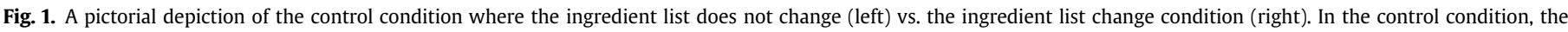

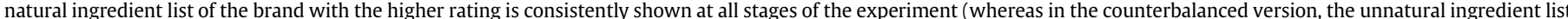

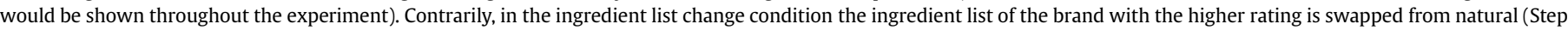
1) to unnatural (at Step 2) (whereas in the counterbalanced version, the swap would be from unnatural to natural).

there were no significant differences between participants in the control and experimental condition or between participants with the natural and unnatural initial ingredient list information in terms of age, gender, education, and employment (all $p$ 's $>.05$ ).

\subsection{Detection rates}

Overall, there were very few participants who had detected the change in ingredient lists as predicted. Observed frequencies indicate that only $16.9 \%$ of all participants from the experimental, ingredient list change condition detected the change. Furthermore, within the general instruction condition $10.7 \%$ of participants detected the change in ingredient list, whereas within the specific instruction condition $23.5 \%$ of participants detected the change. See Table 1 for an overview of the distribution of online detectors and non-detectors. 

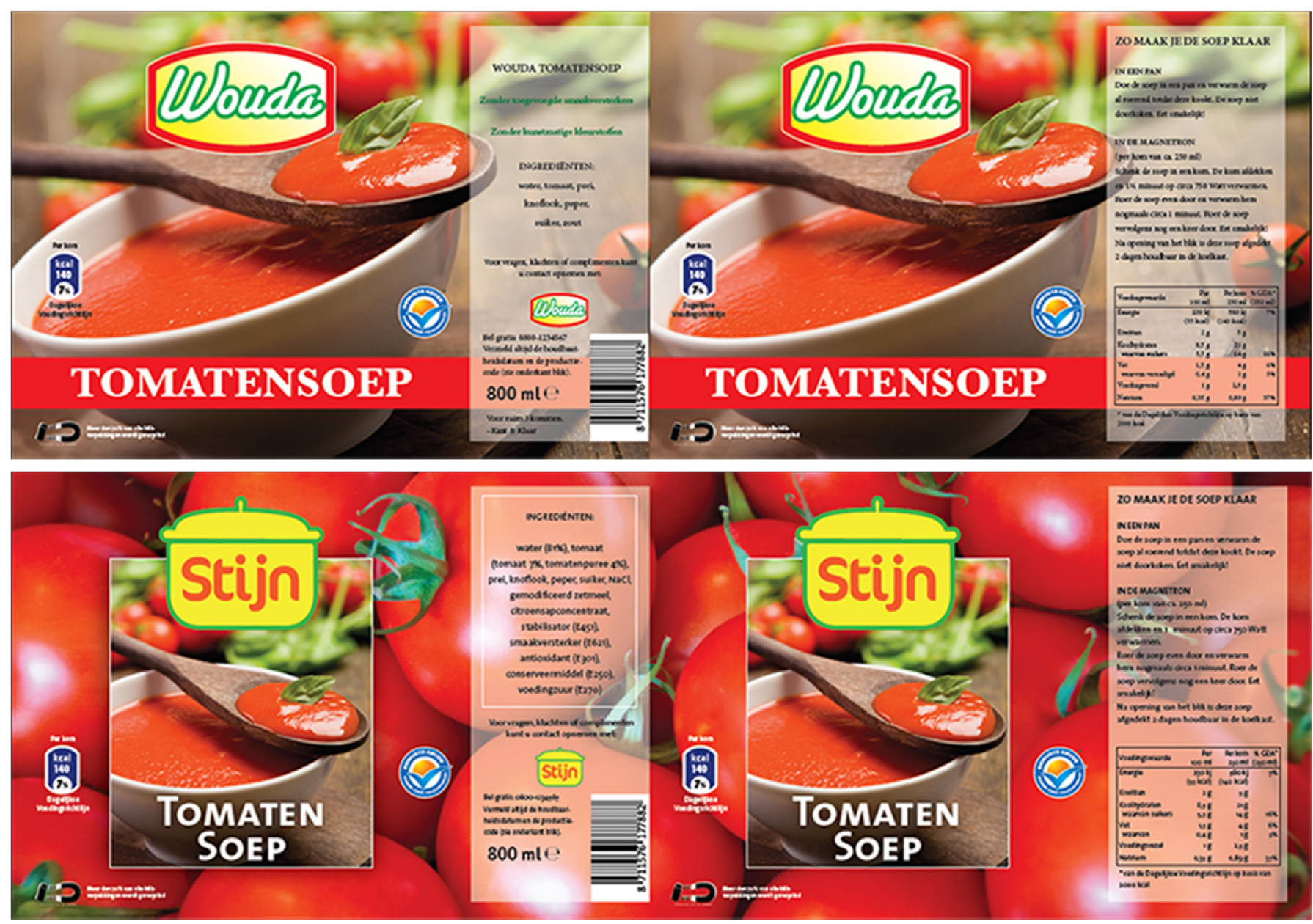

Fig. 2. An example of the package label with a natural ingredient list for Wouda (top), and of a package label with an unnatural ingredient list for Stijn (bottom).
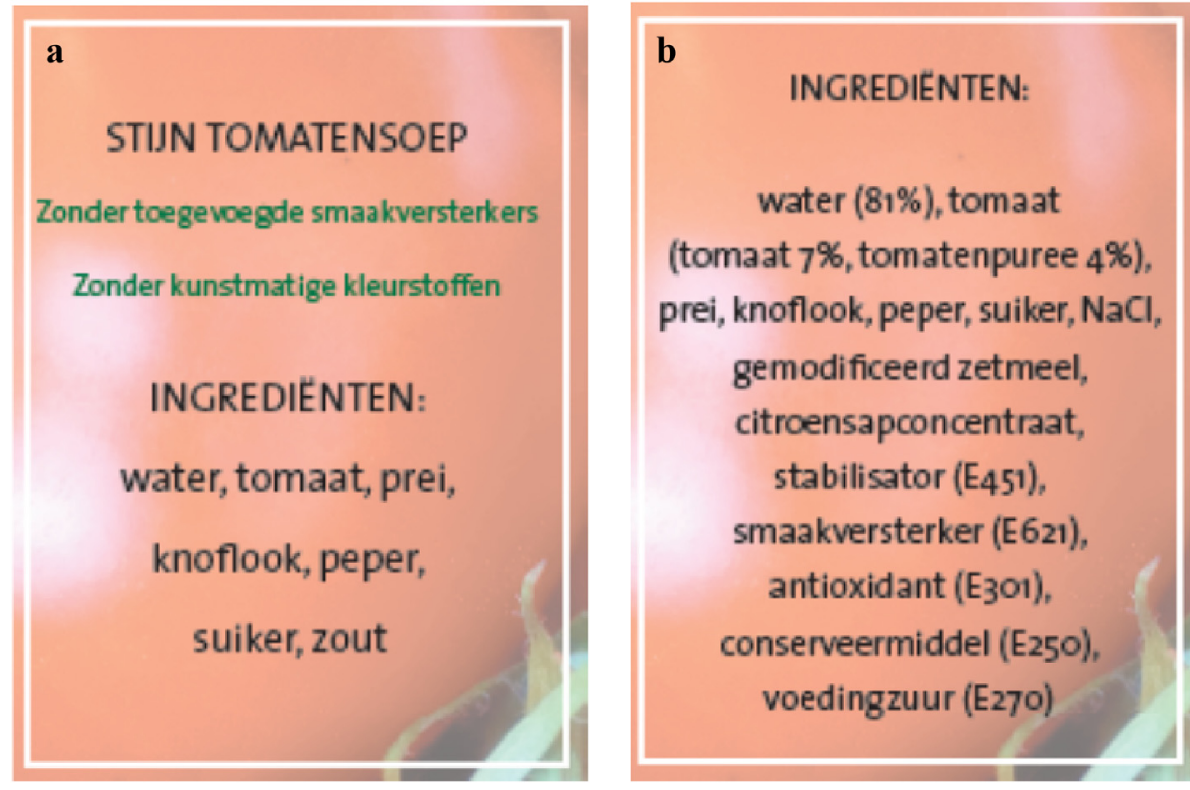

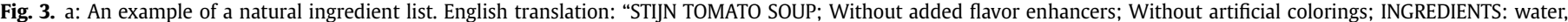

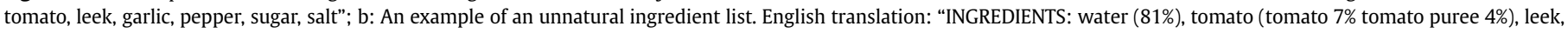
garlic, pepper, sugar, $\mathrm{NaCl}$, modified starch, lemon juice concentrate, stabilizer (E451), flavor enhancer (E621), antioxidant (E301), preservative (E250), food acid (E270). 
Complimenting the observed frequencies that provide preliminary evidence of a higher proportion of online detectors in the specific instruction condition, a logistic regression analysis further tested the hypothesis that predicted detection rates would be higher in the specific instruction condition than in the general instruction condition. Only the participants $(N=308)$ in the change condition were included in the analysis. Additionally, the brand (i.e., Wouda vs. Stijn) of the final product that participants handled during the second stage of the experiment and the ingredient list evaluation order were controlled for in the regression model.

The logistic regression model was statistically significant, $\chi^{2}$ (3, $N=308)=9.60, p=.02$. The model was also $83.1 \%$ correct in predicting online detection. The predictors and the results of the binary logistic regression analyses are presented in Table 2 . In line with hypothesis, results showed that instruction was a significant predictor of detection $(p=.003)$ with an odds ratio of 2.58 . This indicated that participants in the specific instruction condition were 2.5 times more likely to be an online detector compared to participants in the general instruction condition.

Consequently, observed frequencies as well as the results of the logistic regression analysis provide support for hypothesis stating that participants in the specific instruction condition detect a larger proportion of swaps than participants in the general instruction condition.

\subsection{Post-hoc analysis}

\subsubsection{Consumer characteristics}

An exploratory aim of this experiment was to examine whether participants' health concerns, use of information on product packaging and current levels of stress and hunger measured in the final questionnaire would predict their change detection of the ingredient list information. Using Varimax rotation, an exploratory factor analysis revealed six factors with eigenvalues exceeding .6. The suggested factors explained $61.43 \%$ of the variance in the data $(N=492)$, and ultimately one factor was discarded due to a low Chronbach's alpha in the subsequent reliability test of each factor (see Table 3 for an overview). Along with instruction (general instruction vs. specific instruction condition), these five factors including: (1) importance of healthy ingredients, (2) orientation toward quality food indicators, (3) focus on healthy eating, (4) trust in healthiness information, and (5) knowledge of product packaging information, were entered in a binary logistic regression as predictors of detection as the outcome. The logistic model was statistically significant $\chi^{2}(8, N=308)=19.47, p=.013$, and was $83.1 \%$ correct in predicting online detection. However, as presented in Table 4 results indicated that only instruction $[B=.98$; Exp $(B)=2.66, p=.003]$ was a significant predictor of online detection. None of the five factors representing different aspects of consumer characteristics significantly influenced participants' detection of the ingredient list change.

\subsubsection{Referral to ingredient list information}

An additional analysis was conducted to explore whether participants consider the ingredient make-up of the product in justifying their general or naturalness evaluation of the product. During

Table 1

Proportion of online detectors in the general and specific instruction conditions respectively.

\begin{tabular}{ll}
\hline & Proportion of online detections \\
\hline General instruction & $17 / 159$ \\
& $10.7 \%$ \\
Specific instruction & $35 / 149$ \\
& $23.5 \%$ \\
\hline
\end{tabular}

the third stage of the choice-blindness paradigm, participants were asked to explain their overall rating (general instruction condition) or their natural rating (specific instruction condition) of the product as part of the detection assessment. In the condition where the ingredient list changed, 190 participants ignored the ingredient list information when explaining their rating, 58 participants referred to ingredient information but nonetheless did not detect that change. Only 52 participants referred to the ingredient list information and detected the change concurrently. As expected, there were significantly more participants who referred to the ingredient list information in the specific instruction condition, hence also resulting in more detectors, compared to the general instruction condition (see Table 5$), \chi^{2}(2, N=308)=8.85, p=.012$. There was missing information from eight participants in the ingredient list change condition. Additionally, one participant was coded as a retrospective detector as they only disclosed at the end of the experiment that they had noticed, but was uncertain, that there was a change to the ingredient list.

\section{Discussion}

In line with the expectations, our main findings first show that only a low proportion of participants detected the swap of ingredient lists at all. Second, the observation of a higher proportion of detectors in the specific instruction condition (23.5\%) compared to the general instruction condition (16.9\%) compliment the results from the logistic model that instruction condition significantly predicted participants' detection status. These findings are consistent with previous research using the choice-blindness paradigm showing that individuals are generally unaware and do not detect the change when presented back with a choice that was not their own (e.g., Hall et al., 2010; Johansson et al., 2005). Moreover, this implies that a fairly low proportion of participants considered the ingredient list a source of information for a general product evaluation as well as for an evaluation of the naturalness of the product. Finally, our results are particularly interesting because they indicate that consumers do not attend to ingredient list unless specifically directed towards it by a question about 'naturalness'. The additional findings from the post-hoc analyses also support this view, as a greater proportion of participants referred to the ingredient list information and were detectors in the specific instruction condition regarding naturalness, and that besides this naturalness instruction no other consumer characteristics such as health concerns and generic use or consideration of product packaging information predicted detection.

The discrepancy between the often-reported preference for natural products and the here observed lack of attention to ingredient lists could be explained in two different ways. Firstly, the mismatch could be attributed to the characteristics of self-report measures. When engaging in self-report measures consumers may over-report their usage of ingredient information and preference for more natural products in order to present themselves in a positive light that they are critical and healthful agents. The choiceblindness paradigm in the current study avoided the shortcomings of self-report measures and allowed an unbiased measurement of the degree to which consumers attend to and use ingredient list information to evaluate a food product overall and on its naturalness. Thus, the findings could be interpreted such that consumers are less attentive to the 'naturalness' of the ingredients in actual choice-situations than self-reports indicate.

Secondly, it could be that consumers are genuinely concerned with ingredient naturalness, as indicated on self-report measures, but require a specific reminder or cue, such as a question specifically about 'naturalness' as employed in the current study, to guide their behavioral information search to the ingredient list on the 
Table 2

Predictors of online detection (logistic regression).

\begin{tabular}{|c|c|c|c|}
\hline \multicolumn{4}{|l|}{ Dependent variable: Online detection } \\
\hline & $B$ & Sig. ${ }^{\mathrm{a}}$ & $\operatorname{Exp}(B)$ \\
\hline \multicolumn{4}{|l|}{ Nagelkerke R square $=.051$} \\
\hline \multicolumn{4}{|l|}{ Cox \& Snell R square $=.031$} \\
\hline Instruction (base: General instruction) & .947 & .003 & 2.58 \\
\hline Ingredient list evaluation order (base: Natural to unnatural) & -.213 & .491 & .808 \\
\hline Final brand of chosen product (base: Wouda) & .052 & .869 & 1.05 \\
\hline Constant & -2.05 & .000 & .129 \\
\hline
\end{tabular}

a Based on Wald statistic.

Table 3

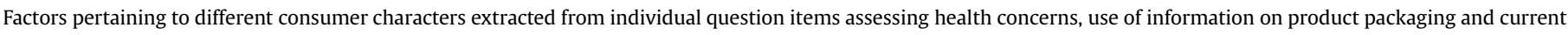
levels of stress and hunger.

Factor 1: Importance of healthy ingredients $(\alpha=.532)$

1. I base my choice for food on health.

2. I base my food for choice on the total amount of calories.

3. The ingredients have no influence oh my choice of food.

4. My purchase considerations are more based on my gut feelings than on careful deliberations.

5. I always look at the ingredients on the label.

6. I use the information on the label to make a decision if I am buying a new product.

7. I use the ingredient information to decide whether I will buy the product.

8. I am interested in ingredient information.

9. Ingredients are important to assess whether the product is healthy if it is unhealthy

Factor 2: Orientation toward quality food indicators $(\alpha=.796)$

1. If a product carries a Fair Trade label I am more inclined to buy it.

2. If a product is organic I am more inclined to buy it.

3. Do you try to eat organic products?

Factor 3: Focus on healthy eating $(\alpha=.705)$

1. Healthy eating is important.

2. How healthy do you think you usually eat?

3. Do you manage toe at healthily?

Factor 4: Trust in healthiness information $(\alpha=\mathbf{. 5 9 8})$

1. If a product carries a health label I am more inclined to buy it

2. If product carries a health label, it is healthier than products without the label

3. I trust that the information represented by the product label is correct

Factor 5: Knowledge of product packaging information $(\alpha=.512)$

1. I understand the information of product packaging.

2. I know what E-number means.

Factor 6: Immediate determinants of purchase ( $\alpha=.354$; discarded due to low Cronbach's alpha)

1. I base my choice for food on taste.

2. I base my choice for food on price

3. I base my choice for food on feelings of hunger.

Table 4

Predictors of online detection (logistic regression).

\begin{tabular}{|c|c|c|c|}
\hline \multicolumn{4}{|l|}{ Dependent variable: Online detection } \\
\hline & $B$ & Sig. ${ }^{a}$ & $\operatorname{Exp}(B)$ \\
\hline \multicolumn{4}{|l|}{ Nagelkerke R square $=.10$} \\
\hline \multicolumn{4}{|l|}{ Cox \& Snell R square $=.06$} \\
\hline Instruction (base: General instruction) & .98 & .003 & 2.66 \\
\hline Ingredient list evaluation order (base: Natural to unnatural) & -.235 & .471 & .79 \\
\hline Final brand of chosen product (base: Wouda) & .009 & .979 & 1.01 \\
\hline Factor 1: Importance of healthy ingredients & .32 & .154 & 1.38 \\
\hline Factor 2: Orientation toward quality food indicators & .228 & .084 & 1.26 \\
\hline Factor 3: Focus on healthy living & .030 & .898 & 1.03 \\
\hline Factor 4: Trust in healthiness information & -.260 & .070 & .77 \\
\hline Factor 5: Knowledge of product packaging information & -.022 & .864 & .98 \\
\hline Constant & -4.137 & .004 & .02 \\
\hline
\end{tabular}

${ }^{\text {a }}$ Based on Wald statistic.

product packaging. This explanation is supported by the finding that detection rates were higher in the specific instruction condition, which may indeed have reminded participants to consider naturalness. Such reminders or cues therefore may provide an opportunity to increase consumers' attention to information they may otherwise overlook in rather mindless product evaluation situations. They could for example come in the form of nudges or labels.

Consumers have a lot of indirect influence in dictating how food policies are regulated and established, as well as how food products are manufactured and marketed. All food additives used in food products are required by the European Food Safety Authority to be extensively tested against health risks, and subsequently identified by respective E-numbers on the ingredient list of the food- 
Table 5

Referral to the ingredient list by non-detectors and online detectors from the general and specific instruction conditions respectively.

\begin{tabular}{|c|c|c|c|}
\hline & Participants who ignored the ingredient list & Non-detectors who referred to the ingredient list & Detectors who referred to the ingredient list \\
\hline \multirow[t]{2}{*}{ General instruction } & $106 / 154$ & $31 / 154$ & $17 / 154$ \\
\hline & $68.8 \%$ & $20.1 \%$ & $11.0 \%$ \\
\hline \multirow[t]{2}{*}{ Specific instruction } & $84 / 146$ & $27 / 146$ & $35 / 146$ \\
\hline & $57.5 \%$ & $18.5 \%$ & $24.0 \%$ \\
\hline
\end{tabular}

packaging label to further inform and reassure consumers (Van Dillen et al., 2003). However, as the findings in our current study show, consumers generally pay less attention to information on ingredient lists than would be expected based on self-reports. This finding suggests that E-numbers as a source of information do not reach the majority of consumers. On the other hand, our findings do not support the idea that 'clean labels', containing a minimum of additives and limited processing, which food manufacturers have increasingly adopted in recent years (Edwards, 2013; Hoogenkamp, 2012), would have a large impact on consumers. Finally, our study also indicates that consumers may require some reminder to attend to the 'naturalness' of ingredients to take this information into account. Despite the fact that the instruction to attend to naturalness improved attention to ingredient lists only for a small proportion of the participants, this finding can be considered a starting point for future research investigating the effectiveness of employing various cues that remind consumers to consider factors they themselves consider important, during actual choice situations. Based on the current results the implementation of subtle cues in the environment may be an effective strategy to shift consumers' attention to information on food packaging they consider relevant.

Besides providing insight into consumers' (in)attention towards ingredient lists, the current study contributes to the literature on choice blindness: whereas the paradigm has mostly been used to demonstrate inconsistencies in people's choices, as well as political and moral attitudes (Hall et al., 2010, 2012, 2013), the current study shows that it can also be a useful strategy to unobtrusively assess consumers' attention to visual components of food products.

Future research is encouraged to develop strategies to understand the (limited) impact ingredient lists have on consumer evaluation and choice of food products. If the aim is to increase the impact of cues in their guidance of consumers' attention to relevant information, either on food packaging or elsewhere (e.g. at specialized websites) more specific studies are needed. The framework used in the current study (choice blindness) may be suited for this, as it does not rely on self-report nor does it alert consumers to aspects of the products they would normally not consider. However, it should be acknowledged that the design of the choice blindness paradigm does not allow for an examination of the cognitive mechanisms that underlie the resulting lack of change detection, and to the best of our best knowledge this has not been examined in previous research. As such, while it is assumed that participants did not notice the change to the ingredient list on the returned product because they had not attended to the ingredient list on the initial product, it could also be plausible that participants did indeed look at the ingredient list information at first, but a lack of thorough processing of the information, a lack of memory of the information, or a failure to use the information subsequently could be accountable for the choice blindness effect. In any case, the implication remains that participants' visual attention to or depth of processing of ingredient list information is not substantial, thereby challenging the notion that consumers highly involve ingredient list information to deduce a product's naturalness. Moreover, to complement our current research methods, future studies could also employ eye tracking as an alternative method to directly assess consumers' visual attention towards ingredient list information on food packaging. Finally, while the current finding of low change detection is consistent and supportive of previous choice blindness studies, it would be beneficial for future research to further examine and pinpoint the cognitive processes that are culprit to the choice blindness effect.

Furthermore, some insight could be drawn from previous literature suggesting consumer's lack of consideration of information on food packaging is not necessarily due to an inability to make use of the information, but rather a lack of motivation (Grunert \& Fernandez-Celemin, et al., 2010; Grunert \&Wills, et al., 2010). It has been acknowledged that consumers do not realize that they make over 200 food-related decisions each day (Wansink \& Sobal, 2007), and that many of these consumption decisions are made mindlessly (Bargh, 2002; Dijksterhuis, Smith, Van Baaren, \& Wigboldus, 2005). In light of this, it would be useful for future research to extend on the current study in examining the implementation of subtle cues to motivate and remind consumers to be more cognizant of information on food packaging that would useful in guiding their purchase decisions. Finally it should be noted that neither behavioral intention nor actual purchasing behavior was measured in this present study. Despite the advantages of instructed product evaluations, the experimental setting does obviously not resemble an actual point of purchase situation very closely. Moreover, previous research has suggested that the reading of ingredient list differs from product to product (Grunert \& Fernandez-Celemin, et al., 2010; Grunert \&Wills, et al., 2010; Nordic Council, 2004), but in this study only one food product was evaluated. A final limitation that should be discussed is the possibility that some of the participants did detect a swap but attributed it to their own wrongful memory rather than an actual inconsistency in what they were presented. Despite taking measures against this possibility by following a four-step detection assessment the possibility cannot be ruled out.

In conclusion this study showed that consumers pay much less attention to ingredient lists than self-reported preferences would suggest, and stresses the limited value of adhering to commonly held beliefs about what ingredient declarations on food products should look like. Cueing considerations of naturalness could be a starting point for increasing consumers' attention to product packaging information they would otherwise neglect.

\section{Financial disclosure}

The study was commissioned by Unilever R\&D Vlaardingen who was involved exclusively in initiating the study design and funding the recruitment of participants for data collection. The funding source was not involved in other aspects of the study. All authors declare no conflicts of interest.

\section{Acknowledgments}

We would like to thank Unilever R\&D Vlaardingen for collaborative efforts and funding support in this research project. Furthermore, we would like to acknowledge Rene Lion for his support and feedback in enhancing the quality of the manuscript. 


\section{Appendix}

Sample Product Evaluation Form.

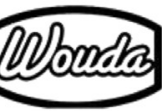

Consumenten survey

Datum

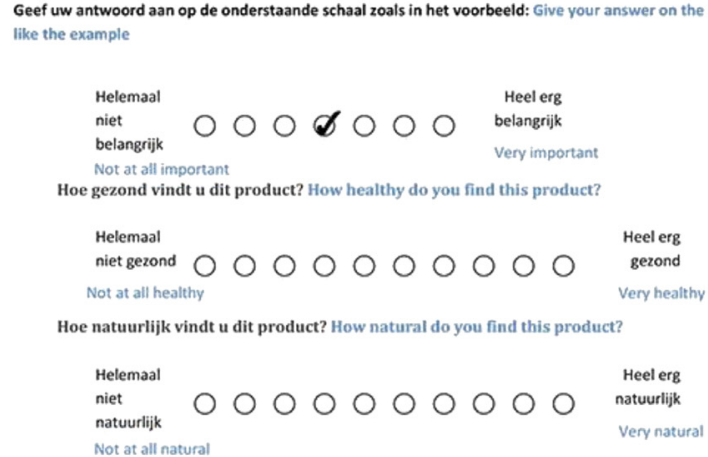

Hoe lekker denkt u dat dit product zal smaken? How tasty do you think this product tastes?

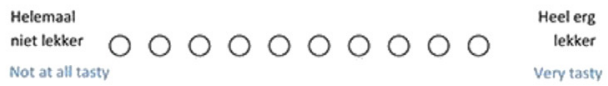

Hoe bekend bent u met het merk van dit product? How familiar are you with the brand of this product?

Helemaal

niet bekend 0000000000

Heel erg

Not at all familiar

bekend

pp

Hoe authentiek vindt $u$ dit product? (een authentiek product is een puur en een eerlijk product dat je met een gerust hart koopt) How authentic do you find this product? (an authentic product is one that is pure and honest)

$$
\begin{aligned}
& \begin{array}{l}
\text { Helemaal } \\
\text { niet } \\
\text { authentiek }
\end{array} \\
& \text { a }
\end{aligned}
$$$$
\text { Not at all authentic }
$$

Hoe vaak gebruikt $u$ deze tomatensoep? How often do you use this tomato soup?

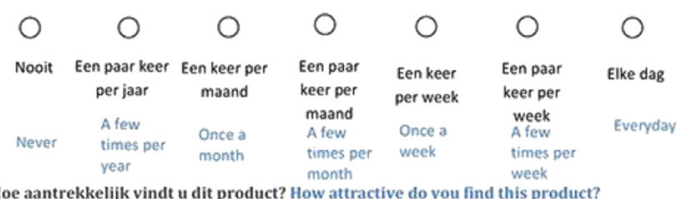

Hoe aantrekkelijk vindt u dit product? How attractive do you find this product?

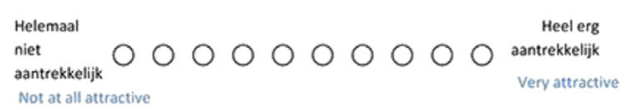

Hoe mooi vindt u de verpakking van dit product? How nice do you find the packaging of this product?

$\begin{aligned} & \text { Helemaal } \\ & \text { niet mooi }\end{aligned}$
Not nice at all

Hoe beoordeelt u deze soep in z'n geheel? How would you rate this soup overall?
Zeer slecht 0000000000

\section{References}

Azjen, I., \& Fishbein, M. (2005). The influence of attitudes on behavior. In D. Albarracin, B. T. Johnson, \& M. P. Zanna (Eds.), The handbook of attitudes (pp. 173-221). Muhwah, NJ: Erlbaum.

Bailis, D. S., Segall, A., \& Chipperfield, J. G. (2003). Two views of self-rated general health status. Social Science \& Medicine, 56, 203-217.

Bargh, J. A. (2002). Losing consciousness: automatic influences on consumer judgment, behavior, and motivation. Journal of Consumer Research, 29(2), 280-285. http://dx.doi.org/10.1086/341577.

Bobe, I. U., \& Michel, M. (2011). Processing and product design for natural food products. In Proceedings of the 11th International congress on engineering and food (pp. 687-688). Athens, Greece: International Congress on Engineering and Food. Available at http://www.icef11.org/content/papers/fpe/FPE1272.pdf.

Bredahl, L. (1999). Consumers' cognitions with regard to genetically modified foods - results of a qualitative study in four countries. Appetite, 34, 343-360.

Dickson-Spillmann, M., Siegrist, M., \& Keller, C. (2011). Attitudes toward chemicals are associated with preference for natural food. Food Quality and Preference, 22(1), 149-156.

Dijksterhuis, G. B. (2004). Transparency: who cares? A user manual for a piece of chocolate. In G. J. Hofstede, H. Schepers, L. Spaans-Dijkstra, J. Trienekens, \& A. Beulens (Eds.), Hide or confide? The dilemma of transparency. 's Gravenhage: Reed business information (pp. 159-164).

Dijksterhuis, A., Smith, P. K., Van Baaren, R. B., \& Wigboldus, D. H. (2005). The unconscious consumer: effects of environment on consumer behavior. Journal of Consumer Psychology, 15(3), 193-202.

Drichoutis, A. C., Lazaridis, P., \& Nayga, R. M., Jr. (2006). Consumers' use of nutritional labels: a review of research studies and issues. Academy of Marketing Science Review, 9.

Edwards, A. (2013). Natural \& clean label trends June 2013. Ingredion Incorporated. As retrieved from event.wavecastpro.com/cleanlabeltrends2013/medialibrary/.

Esomar. (2015). In Codes and guidelines in market research. Retrieved from http:// www.esomar.org.

Evans, G., de Challemaison, B., \& Cox, D. N. (2010). Consumers' ratings of the natural and unnatural qualities of foods. Appetite, 54, 557-563.

Grunert, K. G., Fernandez-Celemin, L., \& Wills, J. M. (2010a). Use and understanding of nutrient information on food labels in six European countries. Journal of Public Health, 18, 261-277.

Grunert, K. G., \& Wills, J. M. (2007). A review of European research on consumer response to nutrition information on food labels. Journal of Public Health, 15, 385-399.

Grunert, K. G. Wills, J. M., \& Fernández-Celemin, L. (2010b). Nutrition knowledge, and use and understanding of nutrition information on food labels among consumers in the UK. Appetite, 55(2), 177-189.

Hall, L., Johansson, P., \& Strandberg, T. (2012). Lifting the veil of morality: choice blindness and attitude reversals on a self-transforming survey. Plos One, 7(9).

Hall, L., Johansson, P., Tärning, B., Sikström, S., \& Deutgen, T. (2010). Magic at the marketplace: choice blindness for the taste of ham and the smell of tea. Cognition, 117, 54-61.

Hall, L., Strandberg, T., Pärnamets, P., Lind, A., Tärning, B., \& Johansson, P. (2013). How the polls can be both spot on and dead wrong: using choice blindness to shift political attitudes and voter intentions. PLoS One, 8(4), e60554. http:// dx.doi.org/10.1371/journal.pone.0060554.

Hebert, J. R., Clemow, L., Pbert, L., Ockene, R. S., \& Ockene, J. K. (1995). Social desirability bias in dietary self-report may compromise the validity of dietary intake measures. International Journal of Epidemiology, 24(2), 389-398.

Holm, L., \& Kildevang, H. (1996). Consumers' views on food quality. A qualitative interview study. Appetite, 27, 1-14.

Hoogenkamp, H. (2012). Naturally cured meat products. Fleischwirtschaft International: Journal for Meat Production and Meat Processing 3, 23-27.

Johansson, P., Hall, L., Sikstroöm, S., \& Olsson, A. (2005). Failure to detect mismatches between intention and outcome in a simple decision task. Science, 310, 116.

Klesges, L. M., Baranowski, T., Beech, B., Cullen, K., Murray, D. M., Rochon, J., et al. (2004). Social desirability bias in self-reported dietary, physical activity and weight concern measures in 8- to 10-year-old African-American girls: results from the girls health enrichment multisite studies (GEMS). Preventive Medicine, $38,78-87$.

Kristiansen, C. M., \& Harding, C. M. (1984). The social desirability of preventive health behavior. Public Health Reports, 99(4).

Lindeman, M., \& Stark, K. (1999). Pleasure, pursuit of health or negotiation of identity? personality correlates of food choice motives among young and middle aged women. Appetite, 33, 141-161.

Malhotra, N. K. (1988). Self concept and product choice: an integrated perspective. Journal of Economic Psychology, 9, 1-28.

McCarthy, M., Brennan, M., Kelly, A. L., Ritson, C., de Boer, M., \& Thompson, N. (2007). Who is at risk and what do they know? segmenting a population on their food safety knowledge. Food Quality and Preference, 18(2), 205-217.

Nordic Council. (2004). Nutrition labelling: Nordic recommendations based on consumer opinions. Copenhagen: Nordic Council of Ministers.

Noussair, C., Robin, S., \& Ruffieux, B. (2001). Do consumers not care about biotech foods or do they just not read the labels? Economics Letters, 75, 47-53.

Reed, A., II, Wooten, D. B., \& Bolton, L. E. (2002). The temporary construction of consumer attitudes. Journal of Consumer Psychology, 12(4), 375-388. 
Rozin, P., Spranca, M., Krieger, Z., Neuhaus, R., Surillo, D., Swerdlin, A., et al. (2004). Preference for natural: instrumental and ideational/moral motivations, and the contrast between foods and medicines. Appetite, 43, 147-154.

Shim, S.-M., Seo, S. H., Lee, Y., Moon, G.-I., Kim, M.-S., \& Park, J.-H. (2011). Consumers' knowledge and safety perceptions of food additives: evaluation on the effectiveness of transmitting information on preservatives. Food Control, 22(7) 1054-1060.

Van Dillen, S. M., Hiddink, G. J., Koelen, M. A., de Graaf, C., \& van Woerkum, C. M. (2003). Understanding nutrition communication between health professionals and consumers: development of a model for nutrition awareness based on qualitative consumer research. The American Journal of Clinical Nutrition, 77(4),
1065S-1072S.

Varela, P., \& Fiszman, S. M. (2013). Exploring consumers' knowledge and perceptions of hydrocolloids used as food additives and ingredients. Food Hydrocolloids, 30, 477-484.

Vermeir, I., \& Verbeke, W. (2006). Sustainable food consumption: exploring the consumer "Attitude - behavioral intention" Gap. Journal of Agricultural and Environmental Ethics, 19, 169-194.

Wansink, B., \& Sobal, J. (2007). Mindless eating: the 200 daily food decisions we overlook. Environment and Behavior, 39, 106-123.

WMO. (2012). Central committee on research involving human subjects. Retrieved from http://www.ccmo.nl/. 